\title{
NiVELES DE PLOMO EN LOS INDIVIDUOS DE LA NECRÓPOLIS TARDORROMANA DE LA C/ VIRGEN DE LA MisERICORDIA (VALENCIA)
}

El objetivo general de este estudio ha sido determinar los contenidos de plomo ( $\mathrm{Pb}$ ) en restos de huesos y suelos de la necrópolis de la cl Virgen de la Misericordia (Valencia). El análisis del plomo se emplea en los estudio de paleopatología para identificar casos de intoxicación por este metal. En nuestro caso una elevada presencia de Pb, no relacionada con los suelos en algunos individuos, se ha asociado a los fragmentos de este metal hallados durante las excavaciones. Verosímilmente la incorporación de plomo en los huesos en concentraciones muy elevadas se ha producido durante el periodo de permanencia de los cuerpos en ataúdes elaborados con este metal.

Palabras claves: plomo, diagénesis, ataúdes de plomo.

\section{LEAD LEVELS IN INDIVIDUALS FROM A LATE ROMAN NECROPOLIS OF VALENTIA}

The general aim of the present study is to determine lead contents in samples belonging to different bones and soils. The samples selected for this study belong to adult individuals from the late Roman necropolis of c/Virgen de la Misericordia discovered in 1992, located in Valencia city centre (Comunidad Valenciana, Spain). In paleopathological studies, lead analysis has been employed to identify toxic concentrations of $\mathrm{Pb}$ in ancient populations. We believe that the higher lead levels detected in bones are related to some lead fragments found at the necropolis. $\mathrm{Pb}$ has probably been incorporated in the bones from lead coffins that contained the corpses.

Key words: lead, diagenesis, lead coffin.

El plomo es un metal utilizado desde finales del IV milenio a.C. (Hong et al. 1994), siendo uno de los elementos que han despertado más atención por parte de los estudiosos a la hora de investigar casos de intoxicación en las poblaciones antiguas. En el mundo antiguo se conocían las consecuencias de las intoxicaciones de plomo ya que la primera fuente de información de envenenamiento procede de Hipócrates, que relacionó los cólicos de un minero como síntoma de intoxicación de plomo y en el s. II a.C. Nicandro, poeta griego, describió los síntomas de intoxicación inducida por una excesiva exposición a este metal (Alexipharmaca, I, 600).
Plinio en su obra ya hablaba de las leyes que limitaban la explotación de las minas de extracción de plomo (Naturalis Historiae XXXIV, 48). Marco Porcio Caton (De re rustica CV), Columella (Rei Rusticae, XII, 20) y Plinio (Naturalis Historiae XIV, 21) daban a conocer cómo en el mundo romano se utilizó ampliamente el plomo en la cocina especialmente para cubrir los contenedores en bronce y cobre para no alterar el sabor de la comida y también como edulcorante en la preparación del vino y del mosto. Plinio (Naturalis Historiae XXXIV, 50) avisaba de la toxicidad de las exhalaciones del plomo calentado, afirmando que el agua presente cerca de las minas 
aportaba serios daños a la salud. Finalmente Vitrubio $(D e$ architectura, VIII, 3) criticaba el empleo de las tuberías de plomo, argumentando que las de cerámica eran mas económicas y menos dañinas por la salud. El envenenamiento por plomo fue propuesto por Gilfillan como la principal causa de la caída del imperio romano ya que conllevaba una disminución de la natalidad y de la longevidad (Gilfillan 1965) pero esta teoría nunca ha sido confirmada científicamente. Partiendo de las fuentes literarias la determinación de los niveles de plomo en restos humanos de las poblaciones romanas (Waldron 1983) y otras poblaciones antiguas (Aufderheide et al. 1988; Aufderheide 1989; Ericson et al. 1979) ha resultado una línea de investigación muy atractiva para muchos científicos.

Muchos de los trabajos que han intentado determinar concentraciones de plomo en huesos arqueológicos han sido puestos en duda en las últimas décadas y ha habido un consistente esfuerzo por parte de los investigadores para comprender los mecanismos de transición de los elementos químicos (entre los restos óseos y los sedimentos que los rodean) para evitar confusiones entre contenidos biogénicos y diagenéticos. La estructura y composición química de los huesos puede ser modificada postmortem por muchos factores naturales. Las alteraciones en los huesos ocurren a causa de los procesos naturales (erosión, disolución, precipitación, ataque microbiano, substitución mineral e iónica, recristalización e intercambio isotópico); cuando uno o más de estos factores naturales intervienen, comúnmente se habla de diagénesis o procesos diagenéticos. Diferentes autores han tratado de averiguar el alcance de estos procesos en los estudios de reconstrucción de la dieta, migración, patología y, más en general, nuestra comprensión de las antiguas civilizaciones (Gallello et al. 2013; Lambert et al. 1985; Reiche et al. 1999 y 2003; Surovell y Stiner 2001; Trueman et al. 2004; Tütken y Vennemann 2011; Zapata et al. 2006). A raíz de esto, algunos trabajos se han dedicado al análisis de metales pesados, especialmente del plomo, para estudiar la exposición de las poblaciones antiguas y determinar el grado de contaminación postmortem como consecuencia de los procesos diagenéticos en los restos óseos analizados (Arnay-De-La-Rosa et al. 1997; Budd et al. 1998; González-Reimers et al. 2003; Martínez-García et al. 2004).

El objetivo general de este trabajo ha sido determinar los contenidos de plomo $(\mathrm{Pb})$ en huesos y sedimentos de la necrópolis tardorromana de c/ Virgen de la Misericordia de la zona céntrica de la ciudad de Valencia (España) (fig. 1), descubierta durante una intervención arqueológica de salvamento entre los años 1992-1993 (Roselló Mesquida y Ruiz Val 1996). El periodo cronológico del espacio funerario abarca desde el s. I a.C. hasta principios del IV d.C.

La determinación del plomo en los restos esqueléticos se ha realizado mediante la espectrometría de emisión óptica de plasma acoplado inductivamente (ICP-OES). El objetivo de este estudio ha sido el desarrollo de un método basado en el sistema de muestreo e interpretación de los resultados para la identificación de muestras de hueso con un perfil elemental de plomo menos afectado por la contaminación de los sedimentos.

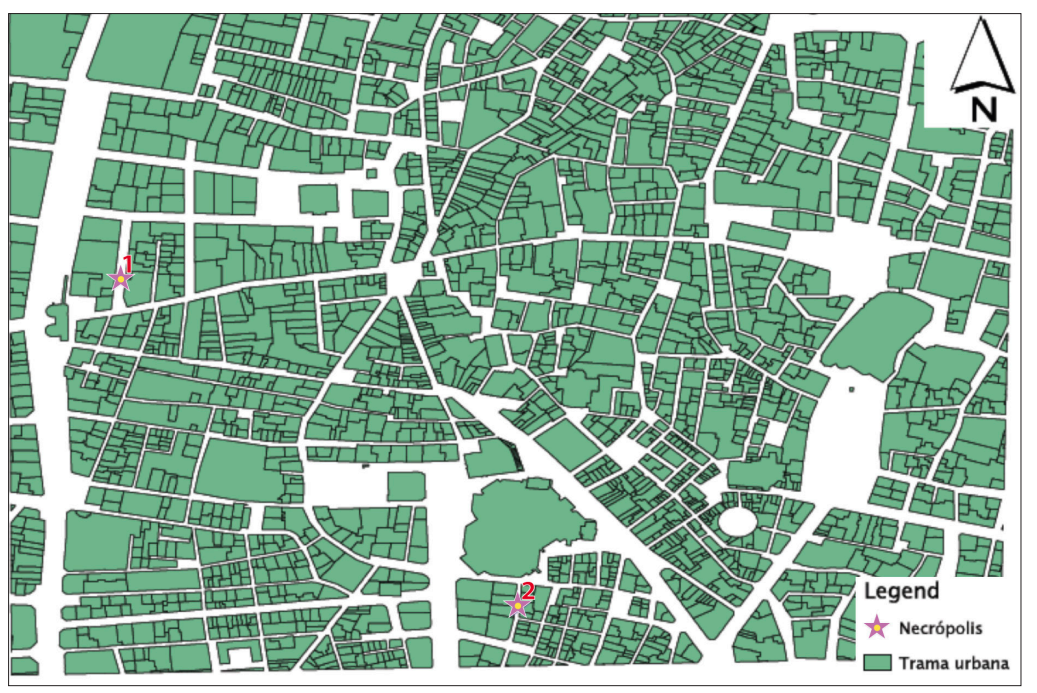

Fig. 1. Valencia Ciudad. Localización de la Necrópolis de la c/ Virgen de la Misericordia (1). 


\section{MATERIAL Y MÉTODO}

Las muestras han sido recogidas en los almacenes del Servicio de Investigación Arqueológica Municipal (SIAM) de Valencia donde se conservaban desde hace unos 15 años sin más información que la contenida en Roselló Mesquida y Ruiz Val (1996) y los informes arqueológicos de la excavación. Los huesos analizados están datados entre los ss. III y IV d.C. y pertenecen a 18 individuos enterrados con el rito funerario de la inhumación. Se ha analizado 87 muestras de huesos de diferentes tipos, capas superficiales de los mismos, sedimentos pegados al hueso y lejanos al mismo. Las muestras provienen de huesos largos (mayoritariamente fémures) o planos (costillas).

La inhumación está documentada en diferentes tumbas. El tipo de tumba más numerosa consiste en una fosa que alberga directamente el cuerpo en un ataúd de madera o en un contenedor cerámico. Hay también fosas con cubierta de tegulae y, en algún caso, fosas con paredes y fondo recubiertas de argamasa y cubierta de tegulae a doble vertiente, tumbas con cista de tegulae, tumbas con semi-cistas de tegulae con cubierta tumular de opus caementicium y tumbas con receptáculo fabricado con paramentos de ladrillo, enlucido y cubierta de tegulae a doble vertiente y tumbas de opus caementicium con planta rectangular, cámara abovedada y cubierta tumular (Roselló Mesquida y Ruiz Val 1996). Las sepulturas son individuales salvo alguna excepción y la orientación predominante es en sentido E-O. Los ajuares hallados están constituidos por piezas cerámicas y alguna de vidrio en las sepulturas más antiguas hasta la segunda mitad del s. I d.C.; a finales de este siglo e inicio del II aparece una única pieza por tumba y a veces una moneda en la boca; a partir de s. III d.C. no se encuentran ajuares y parece desaparecer la costumbre de colocar ofrendas en el interior de las tumbas. Los arqueólogos hallaron pequeños fragmentos de planchas de plomo que interpretaron como la posibilidad de que alguna tumba contuviera un ataúd de este metal que habría sido expoliada en épocas posteriores (Roselló Mesquida y Ruiz Val 1996), pero se desconoce cuál de los individuos estudiados habría sido sepultado en este tipo de ataúd.

\section{ANÁLISIS QUÍMICO}

Las muestras se tomaron de diferentes huesos de los 18 individuos (fig. 2). Las 87 muestras analizadas se subdividen en 12 muestras de fémur, 19 de la parte compacta de la costilla, dos de tibia, una de húmero, nueve de la

\begin{tabular}{|c|c|c|c|c|c|c|c|}
\hline $\mathbf{U E}$ & H.L. & S.H.L. & T.H.L. & C. & S.C. & T.C. & T.H.L.C. \\
\hline $\mathbf{1 0 1 2}$ & N.P. & N.P. & N.P. & $128 \pm 2$ & $93.1 \pm 0.3$ & $17.5 \pm 1.8$ & N.P. \\
\hline $\mathbf{1 3 9 4}$ & N.P. & N.P. & N.P. & $11.4 \pm 2$ & $18.6 \pm 1$ & $16.4 \pm 1$ & N.P. \\
\hline $\mathbf{1 2 9 6}$ & N.P. & N.P. & N.P. & $35.9 \pm 9.6$ & $26.9 \pm 0.6$ & $14.3 \pm 1.3$ & N.P. \\
\hline $\mathbf{1 1 0 2}$ & $34.4 \pm 3.3$ & $48.9 \pm 3.6$ & N.P. & $84.1 \pm 2.4$ & $72.5 \pm 6.1$ & $12.3 \pm 1.7$ & N.P. \\
\hline $\mathbf{1 7 6 3}$ & $28.4 \pm 2.3$ & $62.8 \pm 4.5$ & $64.6 \pm 1.3$ & $21.2 \pm 1.1$ & $44.3 \pm 3.1$ & $16 \pm 1$ & N.P. \\
\hline $\mathbf{1 0 3 8}$ & $110.5 \pm 2$ & $118.6 \pm 4.7$ & $22.8 \pm 2.3$ & $133.1 \pm 3.6$ & $112.8 \pm 3.6$ & $24.2 \pm 0.2$ & N.P. \\
\hline $\mathbf{1 5 9 8}$ & $68.5 \pm 1.7$ & $140.4 \pm 2.8$ & $28.1 \pm 0.9$ & $63.4 \pm 2.8$ & $74.1 \pm 3.2$ & $18.5 \pm 2.5$ & N.P. \\
\hline $\mathbf{1 6 4 4}$ & LOD & $2 \pm 2$ & N.P. & $1.8 \pm 2$ & $6.2 \pm 0.8$ & $7.7 \pm 1.3$ & N.P. \\
\hline $\mathbf{1 4 5 7}$ & $12.7 \pm 0.7$ & $19.9 \pm 0.7$ & $12.4 \pm 0.9$ & $23 \pm 1.4$ & $24 \pm 1.3$ & $11.9 \pm 1$ & N.P. \\
\hline $\mathbf{1 0 8 9}$ & $43 \pm 2.7$ & $61.6 \pm 1.9$ & $12.9 \pm 0.014$ & $61.5 \pm 2.7$ & $55 \pm 1.3$ & $13.3 \pm 0.9$ & N.P. \\
\hline $\mathbf{1 0 8 7}$ & $24.2 \pm 0.7$ & $63 \pm 0.2$ & $19.2 \pm 0.8$ & $149.6 \pm 1.7$ & $99.7 \pm 1.4$ & $18.5 \pm 0.5$ & N.P. \\
\hline $\mathbf{1 3 9 7}$ & N.P. & N.P. & N.P. & $14.5 \pm 0.7$ & $14.9 \pm 0.5$ & $9.5 \pm 0.9$ & N.P. \\
\hline $\mathbf{1 2 7 5}$ & $56.5 \pm 1.4$ & $63 \pm 2.6$ & N.P. & $61.6 \pm 1.9$ & $88.1 \pm 1.6$ & N.P. & $22.2 \pm 0.6$ \\
\hline $\mathbf{1 4 3 5}$ & $11.1 \pm 0.4$ & $15.9 \pm 1.2$ & N.P. & $15.1 \pm 0.2$ & $19 \pm 1.6$ & N.P. & $10.8 \pm 0.4$ \\
\hline $\mathbf{1 0 8 4}$ & $38.3 \pm 2.1$ & $48.3 \pm 1.5$ & N.P. & $38.7 \pm 2.8$ & $59.2 \pm 1.6$ & N.P. & $39.5 \pm 1.5$ \\
\hline $\mathbf{1 2 2 6}$ & $3.9 \pm 1.1$ & $17.4 \pm 0.2$ & N.P. & $119.93 \pm 0.11$ & $165.1 \pm 3.1$ & N.P. & N.P. \\
\hline $\mathbf{1 1 7 9}$ & $26.8 \pm 0.1$ & $39.4 \pm 2.3$ & N.P. & $42.3 \pm 2.2$ & $42.1 \pm 4.6$ & N.P. & $10.3 \pm 2.7$ \\
\hline $\mathbf{1 5 5 7}$ & $49 \pm 1.2$ & $87.7 \pm 1$ & N.P. & $113.07 \pm 0.5$ & $119.3 \pm 1.4$ & N.P. & $57.3 \pm 1.2$ \\
\hline
\end{tabular}

Fig. 2. Contenido en plomo de cada muestra expresados en $\mu \mathrm{g} / \mathrm{g}$ con sus correspondientes desviaciones estándares. Limite de detección (LOD). Unidad Estratigráfica (UE), huesos largos (H.L.), superficie de los huesos largos (S.H.L.), tierra huesos largos (T.H.L.), costillas (C.), superficie costillas (S.C.), tierra costillas (T.C.), tierra huesos largos y costillas (T.H.L.C), ninguna muestra presente (N.P.). 
parte superficial del fémur, 17 de la superficie de la parte compacta de la costilla, dos de la parte superficial de tibia y una de la parte superficial de húmero. En el caso de las tierras se han analizado cuatro muestras de sedimentos que estaban directamente en contacto con los fémures, 13 de sedimentos en contacto con las costillas, uno de sedimento en contacto directo con la tibia, una de sedimento en contacto con el húmero y cinco de sedimentos de la excavación. La toma de muestras se ha realizado en dos fases. En la primera fase, se ha muestreado el hueso con un instrumento de corte. Se ha recogido una muestra de fémur, tibia y/o costilla de los individuos estudiados. Todos los huesos estaban sin lavar y por esto los sedimentos recogidos se encontraban en contacto directo con los huesos. Una vez separados estos sedimentos se pasa a la segunda fase. En todas las muestras de huesos se ha rascado con un bisturí la primera capa en contacto con los sedimentos. En todos los huesos se raspan las capas interna y externa siempre por medio de un bisturí. El raspado de ambas capas se junta en un única muestra. En el caso de las costillas, al tener un menor porcentaje de hueso cortical y mayor de hueso esponjoso son más friables y se parten sagitalmente para permitir que se rasquen las partes internas y externas del mismo modo que los huesos largos.

Para poder ser medidas en el ICP-OES las muestras de hueso y tierra han sido preparadas siguiendo un protocolo que consta de varias etapas. Primero, las muestras han sido incineradas en una mufla para facilitar el proceso de mineralización con el siguiente programa de etapas de temperatura: I) $30 \mathrm{~min}$ a $150{ }^{\circ} \mathrm{C}$; II) $1 \%$ min hasta $450{ }^{\circ} \mathrm{C}$; III) $24 \mathrm{~h}$ a $450{ }^{\circ} \mathrm{C}$ y IV) $30^{\circ} \mathrm{C}$. Sucesivamente, las muestras han sido pulverizadas y homogenizadas con un mortero de ágata. El método de digestión y la dilución desde la digestión principal ha sido desarrollado para obtener reproducibilidad, comparación de los resultados y compatibilidad con la sensibilidad del método analítico. El método de digestión ha consistido en añadir $1.5 \mathrm{ml} \mathrm{HCl} \mathrm{y}$ $1,5 \mathrm{ml} \mathrm{HNO}_{3}$ con $0,5 \mathrm{~g}$ de muestra (huesos y sedimentos) y dos blancos: muestras sin matriz que contienen el mismo volumen de ácidos de las otras muestras y se preparan de la misma manera. Los blancos se emplean para determinar posibles contaminaciones de las muestras que se pueden experimentar durante el proceso analítico. Durante el proceso de digestión se han empleado tubos calentados en baño maría a $100^{\circ} \mathrm{C}$ durante $40 \mathrm{~min}$. Sucesivamente las soluciones digeridas han sido trasladadas a tubos de plástico de $15 \mathrm{ml}$, enrasando hasta $15 \mathrm{ml}$ con agua ultra purificada. Esta solución ha sido utilizada para medir el $\mathrm{Pb}$. Para la preparación de los patrones, se ha empleado una solución de $\mathrm{Pb}$ de $100 \mu \mathrm{g} / \mathrm{ml}$. Para obtener una solución, la preparación de los patrones de la curva de calibración se han empleado tubos de plástico de $50 \mathrm{ml}$ añadiendo $5 \mathrm{ml}$ de $\mathrm{HNO}_{3}, 5 \mathrm{ml}$ de $\mathrm{HCl}$, y los correspondientes volúmenes de solución de patrones hasta llegar a un volumen de $50 \mathrm{ml}$ con agua ultra purificada. Se ha empleado un rango de concentración entre 0 a 20 $\mu \mathrm{g} / \mathrm{g}(0$ - 0,1 - 0,2 - 0,5 - 1 - 2 - 5 - 10 - $20 \mu \mathrm{g} / \mathrm{g})$. Para evaluar la exactitud del método analítico se han utilizado las muestras certificadas Bone ash NIST 1400 (hueso) y GBW07408 (suelo), se han utilizado como material certificado siendo sometidas al mismo tratamiento que las muestras objeto de estudio. El renio (Re) ha sido empleado como patrón interno. Todos los patrones empleados han sido adquiridos a Sharlab S.L. (Barcelona, España). Una vez preparados, muestras y patrones han sido analizados en un ICP-OES Perkin Elmer 5300 DV (Norwalk, CT, USA).

\section{RESULTADOS Y DISCUSIÓN}

\section{DETERMINACIÓN DE LA COMPOSICIÓN DE Pb}

Una vez preparadas las muestras como se describe en la metodología, se ha llevado a cabo el análisis en ICPOES, donde ha sido analizado el contenido en $\mathrm{Pb}$. Para poder comparar los patrones y las muestras, las unidades de medida se expresan en microgramos/gramos $(\mu \mathrm{g} / \mathrm{g})$. Las 87 muestras analizadas hacen referencia a unidades estratigráficas (UUEE) correspondiendo cada unidad a un individuo. En la figura 1 pueden verse los resultados de los huesos largos (H.L.), parte superficial de los huesos largos (S.H.L.), tierra de los huesos largos (T.H.L.), costillas (C.), parte superficial de las costillas (S.C.), tierra de la costilla (T.C) y los resultados de la tierra de los huesos largos y de las costillas juntas (T.H.L.C.), finalmente las muestras no presentes se indican en la tabla con (N.P.). En las unidades estratigráficas hay indicios que pueden sugerir interpretaciones y reconstrucciones de tipo tafonómico en los huesos.

Los resultados de las concentraciones de $\mathrm{Pb}$ obtenidas en la UE 1012 muestran unos valores en la costilla ligeramente superiores a la parte superficial de la misma y los niveles del suelo son los más bajos de este grupo de muestras; en este caso, los procesos diagenéticos parecen haber afectado solo parcialmente a la costilla, 
pero a causa de la falta de muestras de hueso largo, las interpretaciones no son concluyentes.

Las UUEE 1394, 1296 y 1397 (fig. 2) presentan resultados de costilla, parte superficial y sedimentos de la misma muy similares y, en este caso, las muestras parecen afectadas por la diagénesis.

Los individuos 1102 y 1763 también sufren los procesos diagenéticos; el hueso largo presenta concentraciones superiores de $\mathrm{Pb}$ en la parte superficial del mismo, en la costilla los valores son similares a la parte superficial, el sedimento mantiene valores ligeramente más bajos excepto en el hueso largo de la UE 1763 que contiene las concentraciones más elevadas en este individuo.

El individuo 1038 contiene valores muy elevados de plomo en el hueso largo y parte superficial y en la costilla y parte superficial de la costilla, siendo los valores del sedimento claramente más bajos. En este caso, siendo los valores de las partes superficiales muy similares a los de las partes más internas, podrían existir procesos diagenéticos no relacionados con los suelos.

En el individuo 1598 con contenidos más bajos de $\mathrm{Pb}$ se presentan las mismas dinámicas que en el individuo 1038 (siendo los valores de las partes superficiales de los huesos muy similares a los de las partes internas y las concentraciones en las tierras más bajas).

Las UUEE 1644 y 1457 parecen afectadas por los procesos diagenéticos siendo los valores de las tierras y de los huesos muy similares.

Los individuos 1089, 1087 y 1275 (fig. 2) tienen un comportamiento muy similar en la distribución de las concentraciones de $\mathrm{Pb}$. Las partes superficiales de huesos largos y costillas difieren: los primeros son más reducidos y los segundos más elevados que los valores encontrados en sus respectivos suelos de contacto.

En las UUEE 1435 y 1084 (fig. 2) los valores en tierras y huesos son muy similares y en este caso también debido, probablemente, a procesos diagenéticos.

En la UE 1226 (fig. 1) no hay valores de suelo y en los huesos hay valores superiores en la parte superficial de huesos largos y costillas que en sus partes internas. En la costilla de este individuo los valores son elevados y la superficie tiene la concentración más alta de todo el conjunto de muestras $(165 \mu \mathrm{g} / \mathrm{g})$.

En el individuo 1179 no hay mucha diferencia entre huesos, sus partes superficiales y tierra debido a la acción de los procesos diagenéticos.

En el conjunto de muestras de la UE 1557, los valores de $\mathrm{Pb}$ son más elevados en las partes internas de los huesos que en la parte superficial y la tierra tiene concentraciones más elevadas que la parte interna del hueso largo, en los huesos largos también se observa el acción de los procesos diagenéticos pero en la costilla esto no es muy evidente siendo los valores bastante más elevados que en la tierra.

\section{VALORES DE Pb NO CONTAMINADOS POR LOS SEDIMENTOS}

En las figuras 3 a 6 se muestran los individuos que presentan las más elevadas concentraciones de $\mathrm{Pb}$ en los huesos (UUEE 1012, 1038, 1089 y 1087) y de los cuales se han obtenidos los valores de huesos, partes superficiales y tierras.

En la UE 1012, se aprecian elevadas concentraciones de plomo, sobre todo, en la costilla $(128,28 \mu \mathrm{g} / \mathrm{g}) \mathrm{y}$ en la capa superficial de la misma $(93,09 \mu \mathrm{g} / \mathrm{g})$; al contrario, la tierra mantiene valores más bajos de $\mathrm{Pb}(17,54$ $\mu \mathrm{g} / \mathrm{g}$ ) (fig. 3). En este caso, las elevadas concentraciones de plomo en el hueso y la capa superficial del mismo parecen debidas a circunstancias que se produjeron antes del deceso.

La UE 1038 muestra una elevada acumulación de plomo en el fémur $(110,48 \mu \mathrm{g} / \mathrm{g})$ como también en la costilla $(133,12 \mu \mathrm{g} / \mathrm{g})$. Las partes superficiales de fémur y costilla presentan niveles elevados, respectivamente 118,61 y $112,84 \mu \mathrm{g} / \mathrm{g}$. En las tierras los valores son claramente más bajos (22,82 y 24,16 $\mu \mathrm{g} / \mathrm{g}$ ) (fig. 4). En este caso tampoco se observa una contaminación de los sedimentos en los huesos.

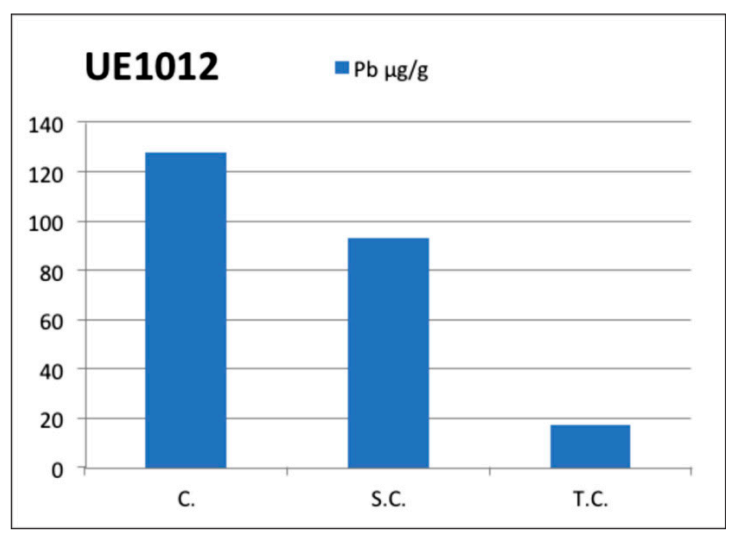

Fig. 3. Resultados de plomo $(\mathrm{Pb})$ expresados en ppm. Unidad Estratigráfica (UE) 1012. Costilla (C.), superficie costilla (S.C.), tierra costillas (T.C). 


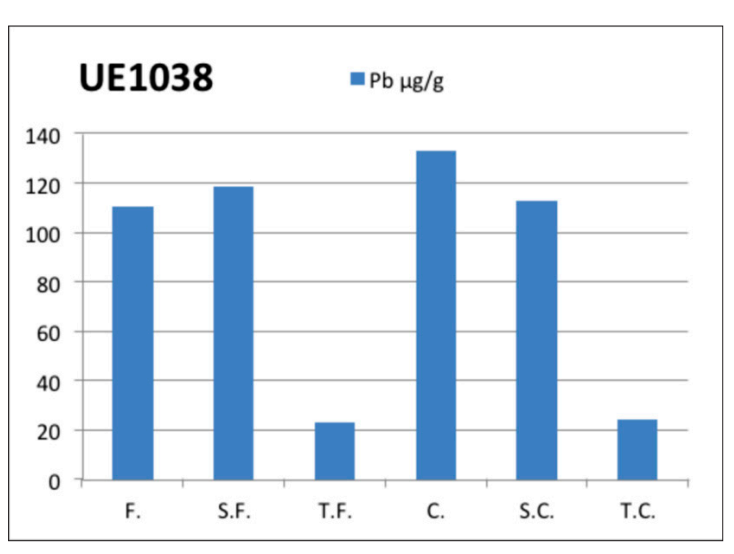

Fig. 4. Resultados de plomo ( $\mathrm{Pb})$ expresados en $\mu \mathrm{g} / \mathrm{g}$. Unidad Estratigráfica (UE) 1038. Femur (F.), superficie femur (S.F.), tierra femur (T.F.), costilla (C .), superficie costilla (S.C.), tierra costillas (T.C.).

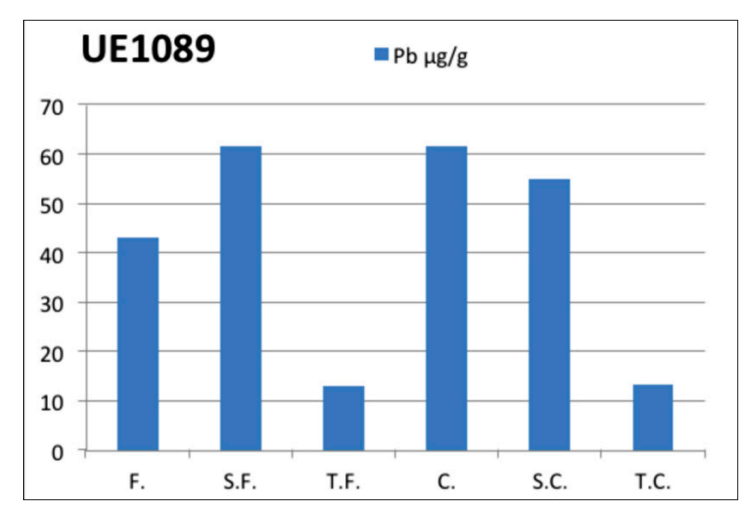

Fig. 5. Resultados de plomo $(\mathrm{Pb})$ expresados en $\mu \mathrm{g} / \mathrm{g}$. Unidad Estratigráfica (UE) 1089. Femur (F.), superficie femur (S.F.), tierra femur (T.F.), costilla (C .), superficie costilla (S.C.), tierra costillas (T.C.).

La UE 1089 indica que las capas superficiales de fémur y costilla contienen las concentraciones más elevadas (respectivamente $61,57 \mu \mathrm{g} / \mathrm{g}$ y $61,45 \mu \mathrm{g} / \mathrm{g}$ ). El fémur contiene $42,99 \mu \mathrm{g} / \mathrm{g}$ y en la parte superficial de la costilla $55,03 \mu \mathrm{g} / \mathrm{g}$. Las tierras tienen valores similares: 12,91 e $13,32 \mu \mathrm{g} / \mathrm{g}$ de $\mathrm{Pb}$ (fig. 5). Tampoco en esta unidad estratigráfica se aprecia el efecto de la diagénesis de la tierra en los huesos.

La UE 1087 muestra concentraciones de plomo más elevadas en la costilla $(149,58 \mu \mathrm{g} / \mathrm{g})$ que en su parte superficial $(99,68 \mu \mathrm{g} / \mathrm{g})$. La capa superficial de la tibia presenta concentraciones menores $(63,03 \mu \mathrm{g} / \mathrm{g})$, la tibia contiene $24,19 \mu \mathrm{g} / \mathrm{g}$, la tierra de la tibia $19,22 \mu \mathrm{g} / \mathrm{g}$ y la tierra de la costilla 18,47 $\mu \mathrm{g} / \mathrm{g}$ de $\mathrm{Pb}$ (fig. 6). En la tibia los valores son similares a los del suelo al contrario que

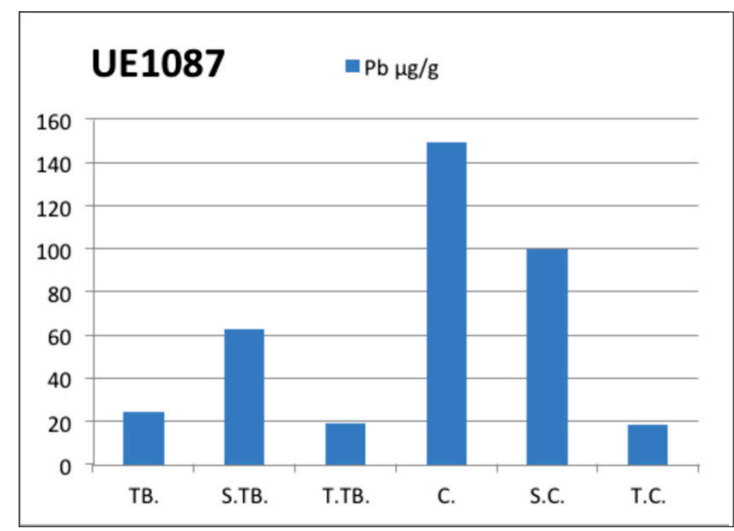

Fig. 6. Resultados de plomo $(\mathrm{Pb})$ expresados en ppm. Unidad Estratigráfica (UE) 1087. Tibia (TB.), superficie tibia (S.TB.), tierra tibia (T. TB.), costilla (C .), superficie costilla (138 S.C.), tierra costillas (T.C.).

la costilla que mantiene valores bastantes más elevados que el suelo.

Resumiendo, muchos individuos en esta población contienen valores elevados de $\mathrm{Pb}(>50 \mu \mathrm{g} / \mathrm{g})$, teniendo en cuenta que los valores considerados no tóxicos en hueso vivo son $<25 \mu \mathrm{g} / \mathrm{g}$ (Needleman et al. 2002). No obstante, en muchos casos se aprecian valores de huesos similares a los de los sedimentos y se podrían relacionar con los efectos de los procesos diagenéticos. En algunos casos (UUEE 1012, 1038, 1089 y 1087) no ha sido posible relacionar la elevada cantidad de $\mathrm{Pb}$ en las diferentes partes de los huesos con la contaminación de los suelos o con los procesos diagenéticos que se originan desde aquéllos. En estos cuatros individuos las muy elevadas concentraciones de $\mathrm{Pb}$ podrían hacer pensar en una intoxicación de plomo ante mortem y así utilizar estas muestras para un estudio paleopatológico. Pero la presencia de fragmentos de $\mathrm{Pb}$ hallados durante las excavaciones (Roselló Mesquida y Ruiz Val 1996), indicando la posible presencia de ataúdes compuestos de este metal, podría explicar el porqué de la similitud de valores entre capas superficiales de los huesos y parte interna de los huesos y la absorción de $\mathrm{Pb}$ durante el periodo de deposición de los cuerpos en este tipo de ataúd. Además estas muestras presentan solamente un aumento de plomo y no de otros elementos metálicos como Cu, Zn, Cd, Cr, Co, Ni y Mn comparándolas con el resto de muestras. Estos resultados pondrían en evidencia las diferencias entre los individuos sepultados en el suelo o en ataúdes de madera y los enterrados en ataúdes de plomo de acuerdo con resultados publicados por algunos autores con anterioridad (Rebôcho et al. 2006). 


\section{CONCLUSIONES}

El análisis en ICP-OES ha sido una técnica adecuada para la determinación de la composición elemental de las muestras. Las conclusiones obtenidas han permitido la identificación de diferencias objetivas entre los distintos tipos de muestras.

Nuestro estudio nos ha permitido determinar los contenidos de plomo del conjunto de muestras de la necrópolis de la c/ de la Virgen de la Misericordia, en uso desde el s. I a.C. hasta el IV d.C. En la mayoría de individuos las concentraciones de $\mathrm{Pb}$ son semejantes a los de los sedimentos por esto creemos que están afectados por los procesos diagenéticos. En algunos casos, sin embargo, no ha sido posible relacionar la elevada cantidad de $\mathrm{Pb}$ en las diferentes partes de huesos con los valores encontrados en los sedimentos. Pero antes de proponer hipótesis de casos de exposición a intoxicación de $\mathrm{Pb}$ ante mortem de estos individuos, consideramos apropiado relacionar la elevada presencia de $\mathrm{Pb}$ con los fragmentos de este metal hallados durante las excavaciones que los arqueólogos responsables de la excavación han interpretado como indicio de la posible presencia de ataúdes de $\mathrm{Pb}$. Verosímilmente, la incorporación de plomo a los huesos en concentraciones muy elevadas se ha producido durante el periodo de deposición de los cuerpos en este tipo de ataúd hecho demostrado anteriormente por otros autores (Rebôcho et al. 2006). Desafortunadamente, las referencias a la presencia de fragmentos de plomo en algunas de las tumbas son genéricas con lo que no podemos relacionarlas, en particular, con ninguna de las tumbas estudiadas por nosotros.

El impacto de los procesos diagenéticos en los huesos ha sido interpretado mediante el análisis de diferentes tipos de hueso del mismo individuo con suelos cercanos al hueso, así como las capas más superficiales de los huesos, empleando el análisis químico en ICP-OES. A través de los análisis de $\mathrm{Pb}$ en restos óseos y empleando una adecuada estrategia de muestreo es posible, en una primera aproximación, asumir la presencia de ataúdes de plomo también sin el hallazgo directos de fragmentos de plomo en las excavaciones.

Consecuentemente creemos que una observación atenta de los contenidos elementales en muestras perteneciente a una determinada unidad estratigráfica puede ayudar a distinguir concentraciones de los elementos condicionados por procesos tafonómicos que se desarrollan independientemente del contexto general debido a las características individuales del ambiente de enterramiento relacionado con las costumbres funerarias y el contexto social de la persona sepultada.

Gianni Gallello

Dept. Prehistòria i Arqueologia/ Dept. de Química Analítica Universitat de València gianga@postal.uv.es

Alessia LarinI Fulvio BARTOLI Francesco Mallegni Dpt. di Biologia Universitá di Pisa planet.express@hotmail.it fbartoli@biologia.unipi.it mallegni.francesco@libero.it

Agustín Pastor García Dept. de Química Analítica Universitat de València agustin.pastor@uv.es

Agustín Diez Castillo Dept. Prehistòria i Arqueologia Universitat de València agustin.diez@uv.es

\section{AGRADECIMIENTOS}

Los autores agradecen la ayuda de Domingo Carlos Salazar (Department of Human Evolution of Max-Planck Institute for Evolutionary Anthropology), Albert Ribera, Pepa Pascual y Josep Vicent Lerma del Servicio de Investigación Arqueológica Municipal (SIAM) de Valencia y del arqueólogo Miquel Roselló por haber proporcionado la documentación arqueológica y las muestras empleadas en este estudio.

\section{BIBLIOGRAFÍA}

ARNAY-DE-LA-ROSA, M.; GONZÁLEZ-REIMERS, E.; VELASCO-VÁZQUEZ, J.; GALINDO-MARTÍN, L.; DELGADO-URETA, L.; SANTOLARIA-FERNÁNDEZ, F.; BARROS-LÓPEZ, N. (1998): Comparison of bone lead in Pre-Hispanic, 18th century and modern population of Tenerife, The Science of the Total Environment 209, 107-111.

DOI: http://dx.doi.org/10.1016/S0048-9697(97)00301-X

AUFDERHEIDE, A. C. (1989): Chemical analysis of skeletal remains, Reconstruction of Life from the Skeleton (M. Y. Iscan y K. A. R. Kennedy, eds.), New York: Alan R. Liss, 237-260. 
AUFDERHEIDE, A. C.; WITTMERS, L. E.; RAPP, G.; WALLGREN, J. (1988): Anthropological applications of skeletal lead analysis, American Anthropologist 90, 931-936. DOI: http://dx.doi.org/10.1525/aa.1988.90.4.02a00110 BUDD, P.; MONTGOMERY, J.; COX, A.; KRAUSE, P.; BARREIRO, B.; THOMAS, R. G. (1998): The distribution of lead within ancient and modern human teeth: Implication for long-term and historical exposure monitoring, The Science of the Total Environment 220, 121-136. DOI: http://dx.doi.org/10.1016/S0048-9697(98)00244-7

ERICSON, J. E.; SHIRAHATA, H.; PATTERSON, C. C. (1979): Skeletal lead concentrations of ancient Peruvians, N. Eng. J. Med. 300, 946-951

DOI: http://dx.doi.org/10.1056/NEJM197904263001703

GAllello, G.; KUligOWSKI, J.; PASTOR, A.; DIEZ, A.; BERNABEU, J. (2013): Biological mineral content in iberian skeletal remains for control of diagenetic factors employing multivariate statistics, Journal of Archaeological Science 40, 2477-2484.

DOI: http://dx.doi.org/10.1016/j.jas.2013.01.022

GILFILLAN, S. C. (1965): Lead poisoning and the fall of Rome, $J$. Occup. Med. 7, 53-60.

GONZÁLEZ-REIMERS, E.; VELASCO-VÁZQUEZ, J.; ARNAYDE-LA-ROSA, M.; ALBERTO-BARROS, V.; GALINDO-MARTÍN, L.; SANTOLARIA-FERNÁNDEZ, F. (2003): Bone cadmium and lead in prehistoric inhabitants and domestic animals from Gran Canaria, The Science of the Total Environment 301, 97-103.

DOI: http://dx.doi.org/10.1016/S0048-9697(02)00299-1

HONG, S.; CANDELONE, J. P.; PATTERSON, C. C.; BOUTRON, C. F. (1994): Green ice evidence of hemispheric lead pollution two millennia ago by Greek and Roman civilizations, Science 23, 1841-1843.

DOI: http://dx.doi.org/10.1126/science.265.5180.1841

LAMBERT, J. B.; SIMPSON, S. V.; SZPUNAR, C. B.; BUIKSTRA, J. E. (1985): Bone diagenesis and dietary analysis, Journal of the Human Evolution 14, 477-482. DOI: http://dx.doi.org/10.1016/S0047-2484(85)80026-9

MARTÍNEZ-GARCÍA, M. J.; MORENO, J. M.; MORENOCLAVEL, J.; VERGARA, N.; GARCÍA-SÁNCHEZ, A.; GUILLAMÓN, A.; PORTÍ, M.; MORENO-GRAU, S. (2004): Heavy metals in human bones in different historical epochs, Science of the Total Environment 348, 51-72.

DOI: http://dx.doi.org/10.1016/j.scitotenv.2004.12.075
NEEDLEMAN, H. L.; MCFARLAND, C.; NESS, R. B.; FIENBERG, S. E.; TOBIN, M. J. (2002): Bone lead levels in adjudicated delinquents a case control study, Neurotoxicology and Teratology 24, 711-717. DOI: http://dx.doi.org/10.1016/S0892-0362(02)00269-6

REBOCHO, J.; CARVALHO, M. L.; MARQUES, A. F.; FERREIRA, F. R.; CHETTLE, D. R. (2006): Lead post-mortem intake in human bones of ancient populations by $109 \mathrm{Cd}-$ basedX-ray fluorescence and EDXFR, Talanta 70, 957-961. DOI: http://dx.doi.org/10.1016/j.talanta.2006.05.062

REICHE, I.; FAVRE-QUATTROPANI, L.; CALLIGARO, T. J. S.; BOCHERENS, H.; CHARLET, L.; MENU, M. (1999): Trace element composition of Archaeological Bones and postmortem alteration in the burial environment, Nuclear Instruments and Methods in Physics Research B 150, 656-662.

DOI: http://dx.doi.org/10.1016/S0168-583X(98)00949-5

REICHE, I.; FAVRE-QUATTROPANI, L.; VIGNAUD, C.; BOCHERENS, H.; CHARLET, L.; MENU, M.(2003): A multi-analytical study of bone diagenesis: the Neolithic site of Bercy (Paris, France), Meas. Sci. Technol. 14, 1608-1619. DOI: http://dx.doi.org/10.1088/0957-0233/14/9/312

ROSELLÓ MESQUIDA, M.; RUIZ VAL, E. (1996). La necropolis occidental de la Valencia romana. Saitabi 46, 147-168.

SUROVELL, T.A.; STINER, M.C. ( 2001): Standardizing Infra-red measures of bone mineral crystallinity : an experimental approach. Journal of Archaeological Science 28, 633-642. DOI: http://dx.doi.org/10.1006/jasc.2000.0633

TRUEMAN, C.N. G.; BEHRENSMEYER,A. K.; TUROSS N.; WEINER, S. (2004): Mineralogical and compositional changes in bones exposed on soil surfaces in Amboseli National Park, Kenya: diagenetic mechanisms and role of sediment pore fluids, Journal of Archaeological Science 31, 721-739. DOI: http://dx.doi.org/10.1016/j.jas.2003.11.003

TÜTKEN, T.; VENNEMANN, T. (2011): Fossil bones and teeth: Preservation or alteration of biogenic compositions?, Palaeogeography, Palaeoclimatology, Palaeoecology 310, 1-8. DOI: http://dx.doi.org/10.1016/j.palaeo.2011.06.020

WALDRON, T. (1983): Lead in ancient bones, Disease in Ancient Man (G. Hart, ed.), Toronto: Irwin Clarke, 172-182.

ZAPATA J.; PÉREZ-SIRVENT, C.; MARTÍNEZ-SÁNCHEZ, M. J.; TOVAR, P. (2006): Diagenesis, not biogénesis: two late Roman skeletal examples, Science of the Total Environment 369, 357-368. DOI: http://dx.doi.org/10.1016/j.scitotenv.2006.05.021 